\title{
Test Methodology to Evaluate the Safety of Materials using Spark Incendivity
}

\author{
Charles Buhler (1), Carlos Calle (2), Sid Clements (3), Mindy Ritz (1), Jeff Starnes (1) \\ (1) Electrostatics and Surface Physics Laboratory, ASRC Aerospace, Kennedy Space Center, FL 32899, USA \\ tel.: 321-867-4861, fax: 321-867-4489, e-mail: BuhleCR@kscems.ksc.nasa.gov \\ (2) Electrostatics and Surface Physics Laboratory, NASA, Kennedy Space Center, FL 32899, USA \\ tel.: 321-867-3274, fax: 321-867-4489, e-mail: carlos.i.calle@nasa.gov \\ (3) Department of Physics and Astronomy, Appalachian State University, Boone, NC 28608, USA \\ tel.: 868-262-2447, fax: 828-262-2049, email: clementsis@appstate.edu
}

\begin{abstract}
For many years scientists and engineers have been searching for the proper test method to evaluate an electrostatic risk for materials used in hazardous environments. A new test standard created by the International Electrotechnical Commission is a promising addition to conventional test methods used throughout industry. The purpose of this paper is to incorporate this test into a proposed new methodology for the evaluation of materials exposed to flammable environments. However, initial testing using this new standard has uncovered some unconventional behavior in materials that conventional test methods were thought to have reconciled. For example some materials tested at higher humidities were more susceptible to incendive discharges than at lower humidity even though the surface resistivity was lower.
\end{abstract}

\section{Introduction}

The NASA Kennedy Space Center (KSC) uses a variety of materials in hazardous spaceport operations. Before materials can be used they must first pass a battery of tests that includes flammability, hypergolic compatibility 
and electrostatics. KSC uses Standard Test Method MMA-1985-79 for evaluating the electrostatic properties of materials [1]. The test, which will be described in detail later, is a triboelectric charge decay test that evaluates the electrostatic properties of the test materials and provides a pass/fail criterion for candidate materials. This one simple test is all that is currently used to fully evaluate the electrostatic properties of spaceport materials.

$\mathrm{KSC}$ uses a variety of hazardous chemicals and flammable materials during normal operations. Safety of the workers is a prime concern. The question of the proper techniques that should be utilized to evaluate electrostatic hazards has been debated for a long time and it is not clear that simple triboelectric testing alone suffices. Therefore, what follows is a new evaluation criteria for the proper selection of materials used during hazardous operations.

\section{Proposed Evaluation Criteria}

Before discussing the proposed test methodology it is important to discuss the relevant factors that can cause incendiary discharges. Incendiary discharges are prominent in materials possessing high surface voltages as a result of significant surface charge. The risk of occurrence of incendiary static discharges depends primarily on the energy liberated which is influenced by the gap size, geometry of the system, mobility of the charge, duration of the discharge, etc. Therefore, an understanding of the electrostatic properties of a material is useful in determining how it might behave in a flammable environment. What follows is a proposed test method based on conventional methods currently used to classify materials (presented here for review) with the addition of two new tests resulting in a vigorous yet realistic evaluation scheme for the sole purpose of identifying materials that could be hazardous in flammable environments.

The first step in evaluating the electrostatic characteristics of a material is to classify the material in terms of its conductivity. If materials are conductive or statically dissipative, then these materials must be electrically bonded to ground in all cases so that any electrostatic charge generated or deposited on them has an electrical path to ground. Depending on the nature of the material and its usage, no further testing is needed if the material can be 
grounded. However, if the materials are insulating, further testing such as charge decay tests should be performed. Charge decay tests are tests in which electrostatic charge is placed on the surface of a material and the time it takes for the charge to dissipate to ground is monitored. Although resistivity tests are performed for just this reason, there are several authors who have claimed that there is no correlation between the surface resistivity of a material and its ability to dissipate charge $[2,3]$. This is because there is a problem with qualifying the charge retention capabilities of a material by a measurement of its resistivity even though this is defined in many standards. Examples for spaceport materials can be seen in Figure 1 below.

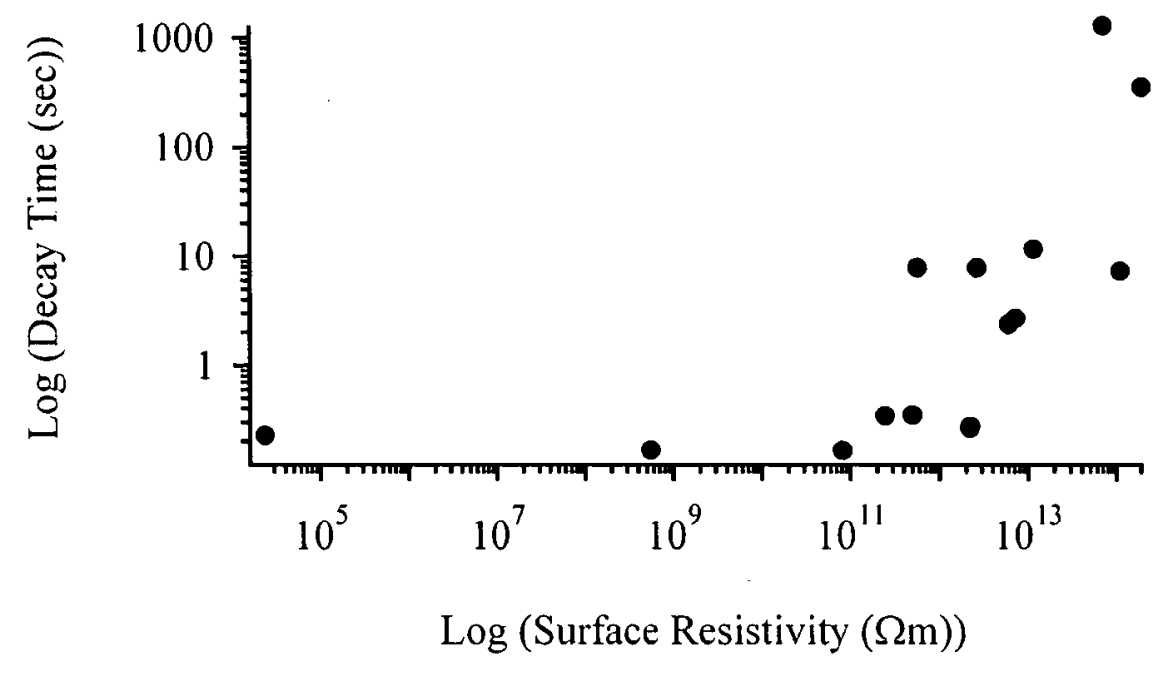

Figure 1: Corona-deposited Charge Decay times versus Surface Resistivity performed for a variety of spaceport materials at $12 \%$ relative humidity.

Two types of charge decay tests should be performed: Triboelectric charging and corona charging. The triboelectric tests simulate the actual charge generation mechanism as seen in the field. An example might be an operator rubbing against other objects or equipment. On the other hand, the amount of charge generated by triboelectric charging is not always repeatable. Depositing a constant amount of charge via a corona source is required. Both tests apply charge to the surface of a material and record the subsequent charge decay or leakage rate.

More importantly, tests should be conducted to see if the charging process itself is capable of producing electrostatic discharges. Electrostatic discharges can be detected in many ways and the most important piece of 
information gathered is whether the discharges are capable of igniting a flammable atmosphere. Accurate measurement of the total energy in a discharge is nearly impossible because the energy may be released in various forms. Discharge energy is released as thermo molecular motion of the air atoms in the form of heat and sound, and as transitional energy of the atomic states in the form of light and electromagnetic waves. Thus capturing all of the forms of energy simultaneously is highly impractical. Ignition also depends on more than total energy; the spatial and temporal distributions of the electrostatic discharge also affect ignition. For example, if the discharge is diffuse or if the discharge occurs slowly, then the energy density may be too low for ignition to occur.

It is difficult to measure how concentrated the electrostatic discharge is and thus it is only feasible to measure the total electrical energy of the discharge. Instead, one can measure a certain characteristic of the energy of interest such as the total amount of charge exchanged. The electrical energy portion of the discharge is well known as $\mathrm{Q}^{2} / 2 \mathrm{C}$, where $\mathrm{Q}$ is the total charge and $\mathrm{C}$ is the capacitance of the gap. Forcing a discharge through a gas to produce ignition is a commonly used method to calculate the Minimum Ignition Energies (MIE) of the gas. Likewise limitations of discharge energies can be measured by immersing the spark-gap in a gas of known MIE. This method for measuring discharge energies serves as the basis for the new Spark Incendivity Test Standard [4]. Charge is placed onto a material using either tribo or corona methods and extracted with a grounded metal probe. As the probe nears the test material it will discharge an area of the charged surface. Gases of known MIE flow between the probe and the charged test sample. If the electrostatic discharge energy is sufficient and the probe is placed greater than the quenching distance, an ignition will occur. If charge placed on the test material is capable of sustaining an incendive discharge the material should not be used.

The user may want to still use the material if it fails the Spark Incendivity Test. This is not unreasonable since the extreme charging method used deposits significant charge on the surface of the test materials which may be considered unrealistic in the field. Therefore a more realistic test would be one which is performed exactly as in the workplace and the conditions replicated. For example, if it is known that a certain material is to be detached from another at high speeds and there is a possibility for a flammable gas to be present, tests should be performed 
inside a controlled environment that simulates this exact process. This test, which on average produces far less electrostatic charge on insulator surfaces than Spark Incendivity, is called "representative testing".

Although the above Spark Incendivity test procedure measures whether extreme amounts of deposited charge is capable of igniting a gas mixture of known MIE, there are limitations to this test method. The prime limitation is that the charge generation occurs before electrostatic discharge measurements are made rather than simultaneously. The test samples are charged in air first before the probe and its gas mixture of known MIE approaches the surface. Since the material is charged in air, the maximum amount of charge on the surface is limited by air breakdown. During separation, triboelectric charging is capable of depositing significant amounts of charge on surfaces well above the breakdown of air but as the surfaces separate, the amount of charge will be limited by gas breakdown if the surface electric fields produced are in excess of $3 \times 10^{6} \mathrm{~V} / \mathrm{m}$. The initial breakdown itself will be in the form of a spark or corona that is not measured using Spark Incendivity. 


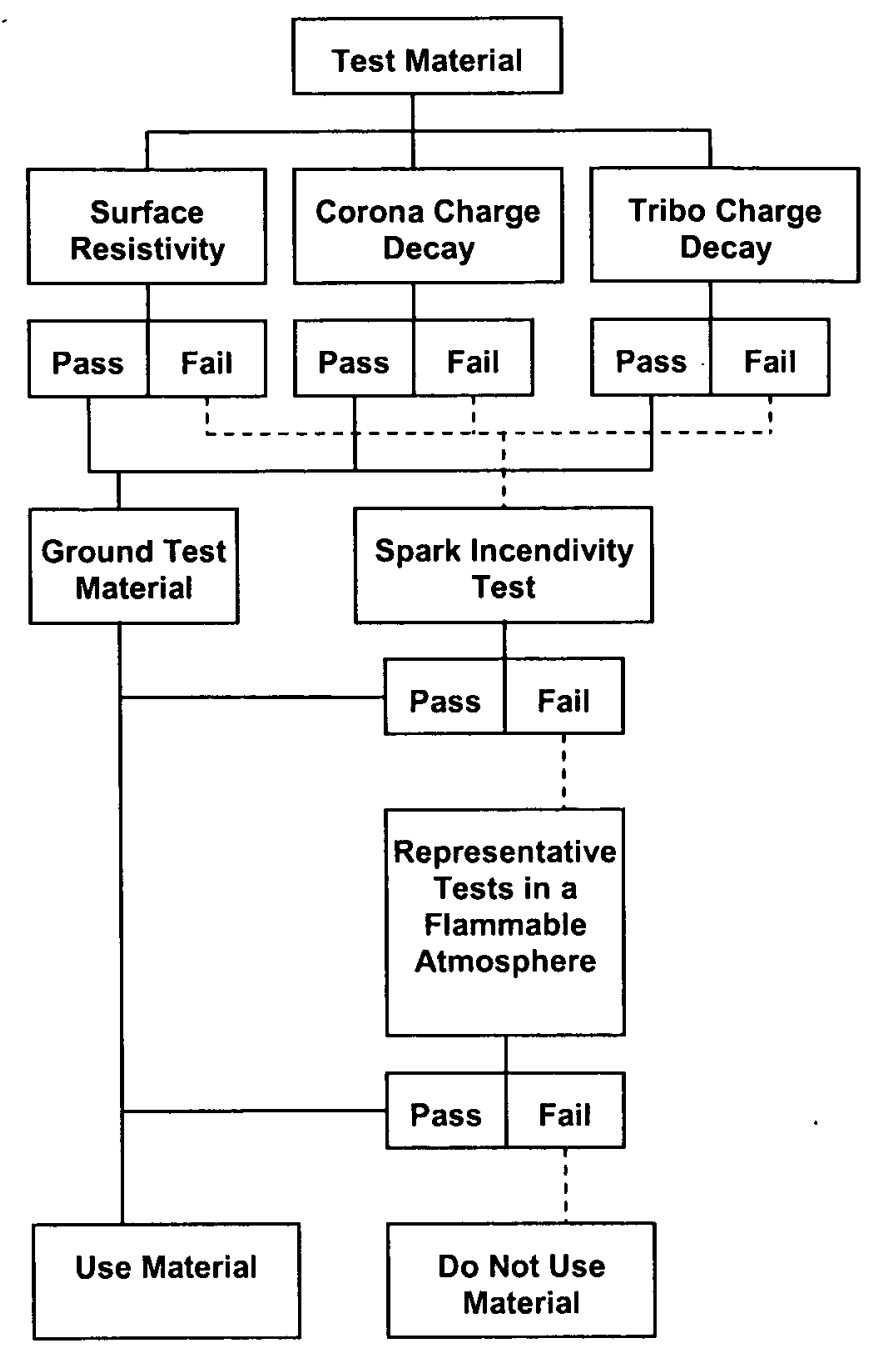

Figure 2: Proposed test method to evaluate the ESD safety of materials used in flammable environments.

Representative testing is capable of capturing this behavior since the triboelectrification and separation occurs in the flammable environment. Thus one might suggest performing representative testing before Spark Incendivity to capture this potentially large discharge event. However Spark Incendivity is a simpler test that deposits significant charge that can be performed quickly while Representative testing requires blast-proof chambers and cannot be performed easily. In the end it is user discretion as to the appropriate methodology to choose based on the material and the environment it is to be used in. For example, clothing materials may be more susceptible to high levels of sustained charge for longer periods of time ideally tested for Spark Incendivity, while a high-speed insulating belt over a grounded metal roller in the presence of a flammable atmosphere would not benefit greatly from a Spark Incendivity test. 
The complete test methodology for evaluating the ESD safety of materials particularly associated with spaceports is shown in Figure 2. The next section describes measurement techniques used to evaluate the proposed test method followed by experimental cases used to evaluate typical spaceport materials.

\section{A. Surface Resistivity}

The first series of tests consisted of measuring the surface resistivity of the materials. Surface resistance measurements are the main test method used in industry to characterize the ESD properties of materials, since it is generally believed that charge deposited onto the surface of a material will "leave" (or decay) easier from a material with lower surface resistance than from a material with high surface resistance. Materials with a surface resistance less than $10^{4} \Omega$ are considered conductive. Materials between $10^{4} \Omega$ and $10^{11} \Omega$ are statically dissipative and materials with a surface resistance above $10^{11} \Omega$ are insulating according to ANSI/ESD standards.

Surface resistivity is the ratio of the DC voltage to the current flowing between two electrodes of specified configuration that contact the same side of the material and is expressed in ohms $(\Omega)$. The surface resistivity tests were performed per the requirements of the ESD Association Standard Test Method ESD STM11.11 [5]. These measurements were taken using a PRS-801 resistance system with an Electro Tech System (ETS) PRF-911 concentric ring resistance probe with up to 100 volts applied between the electrode and ground. The tests require a five pound weight on top of cylindrical electrodes and were conducted at both $20 \% \pm 5 \%$ relative humidity and $50 \% \pm 5 \%$ relative humidity. In order for materials to pass resistivity tests, the surface of the materials must either be conductive or statically dissipative. Otherwise the materials fail the ESD test.

\section{B. Triboelectric Charging}

One method of charging a material is by contact (triboelectric) charging. The Electrostatics and Surface Physics Laboratory at $\mathrm{KSC}$ has been performing triboelectric charging tests of materials for over forty years. The Kennedy Space Center uses Standard Test Method MMA-1985-79 entitled "Standard Test Method for Evaluating Triboelectric Charge Generation and Decay" [1]. This method of evaluating the electrostatic properties of 
materials records the charge decay after the test material is rubbed with either wool or PTFE felt. A device called the ESD Robot used to test materials is shown below in Figure 3.

Two seven-inch square test samples were cut out of each of the stock materials to be tested. They were then mounted onto an aluminum frame and acclimated at $75^{\circ} \mathrm{F}$ for 24 hours prior to testing under relative humidities of $45 \%$ and $30 \%$ compliant with the current KSC standard. The aluminum frames were then mounted on the sample carrier shuttle that operates in two positions. The first position allows a PTFE felt disk or wool disk (about five inches in diameter) to rub against the test sample using five pounds of force. The disk rotates at 400 revolutions per minute for ten seconds to "saturate" the sample with charge. After ten seconds the sample moves into the second position in front of a static detector head that records the surface potential while the aluminum frame is grounded. Shown in Figure 3 is the Keithley model 2501 Static Detector head. However, here we used JCI model 140 Static Monitor with a guard to record surface potential. The current KSC standard dictates that materials pass the ESD test criteria if the surface voltage is below 350 volts after five seconds, otherwise the materials fail.

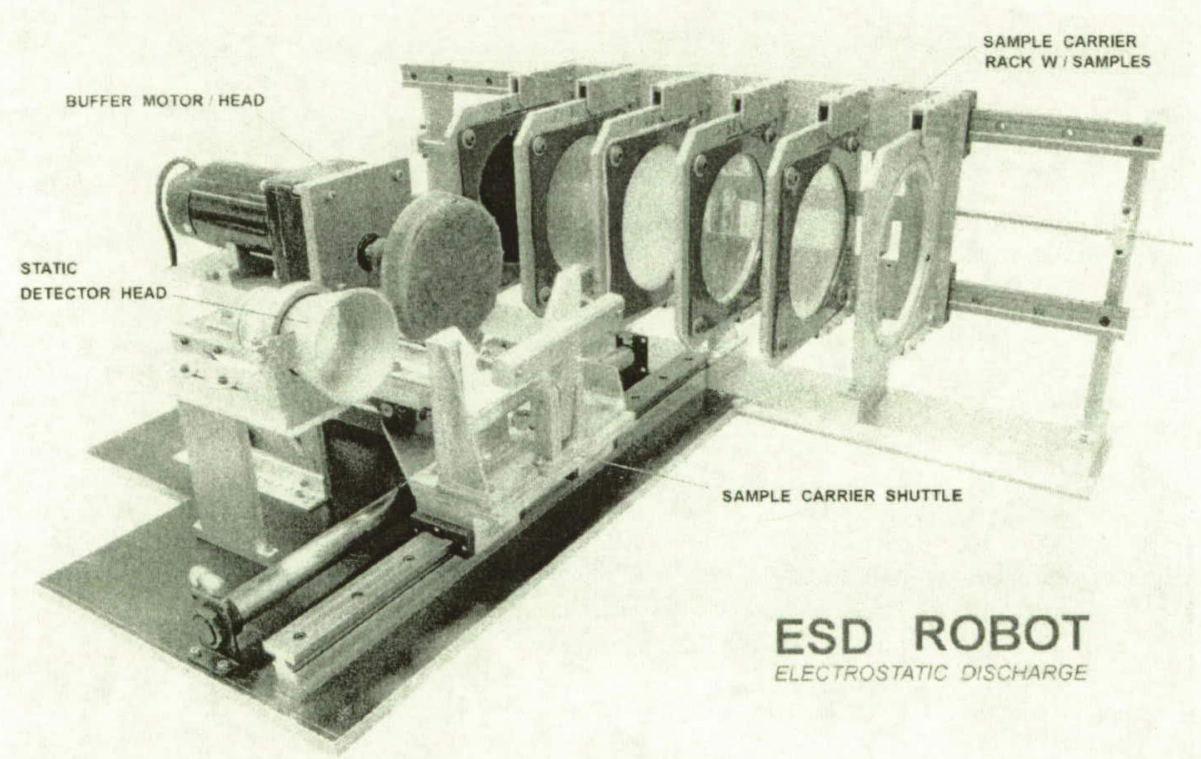

Figure 3: Triboelectric ESD Robot at the NASA Kennedy Space Center. 


\section{Corona Charging}

The next battery of tests conducted on spaceport materials is corona charge decay. Corona charge deposition is chosen because surface charge levels can be deposited in a controlled manner. Tests are performed using a JCI 155v5 Charge Decay Test Unit conforming to British Standard 7506 "Methods for Measurements in Electrostatics" [6]. This device deposits a consistent amount of negative charge onto the surface of a material by ionizing the air molecules with high voltage corona needle points up to $\pm 10,000$ volts [Figure 4]. Materials that have been acclimated at Once mounted, the materials were acclimated at both $20 \% \pm 5 \%$ relative humidity and $50 \% \pm 5 \%$ relative humidity are placed inside the JCI 176 Charge Measurement Sample Support system. The JCI $155 \mathrm{v} 5$ sits on top of a JCI 176 system which has the ability to measure the total amount of charge transferred to the sample [Figure 5]. The total amount of charge transferred to the sample consists of both the charge that "leaves" the surface of the sample and the charge that remains. The samples are mounted between two conducting plates shown in Figure 5.

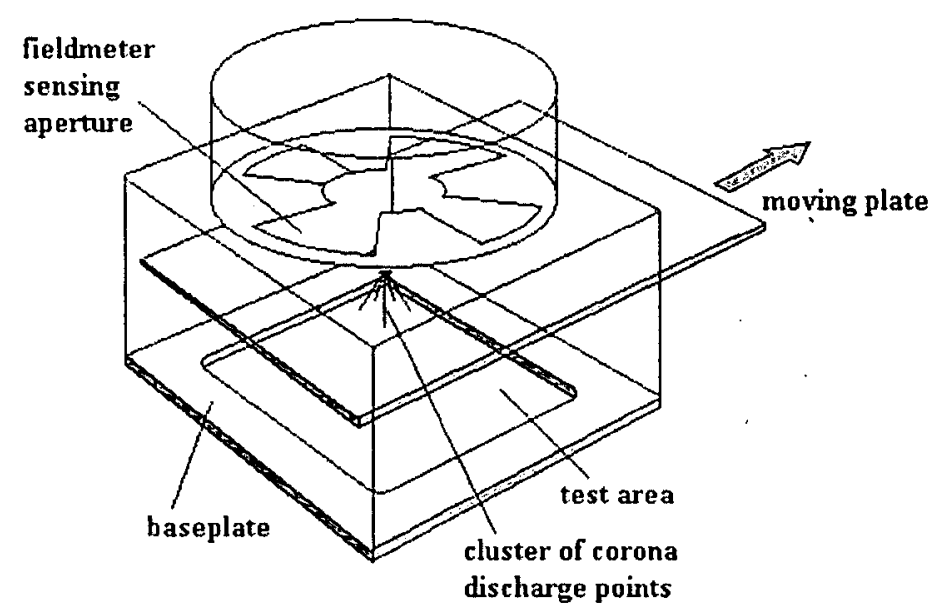

Figure 4: The JCI 155v5 Charge Decay Test Unit. 


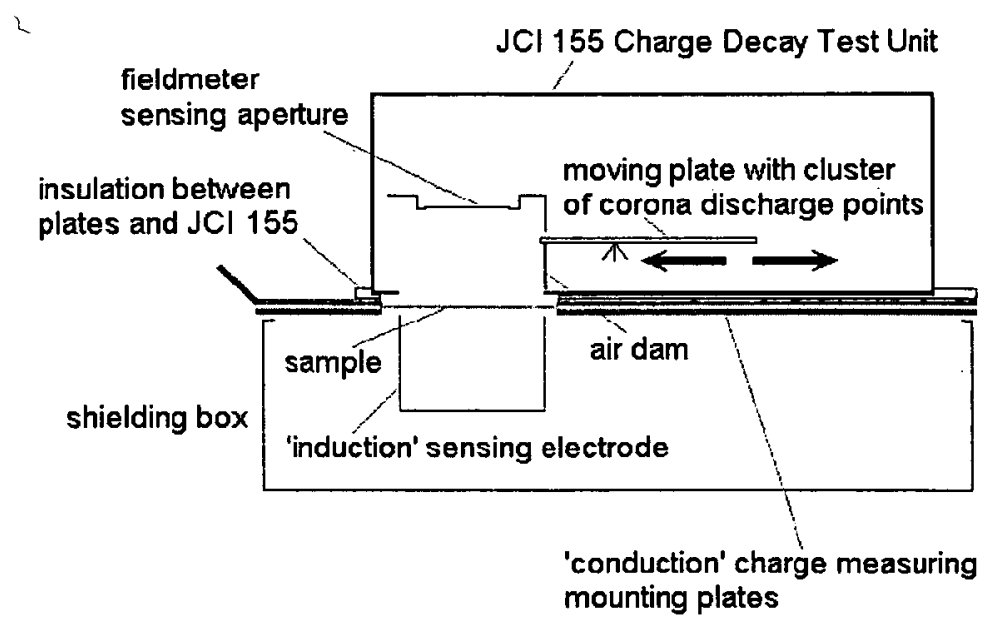

Figure 5: JCI 155v5 sits on top of the JCI 176 Charge Measurement Sample support.

Once the charge is deposited onto the surface of the test material, the plate containing the corona points is retracted (within $20 \mathrm{~ms}$ ) and a fieldmeter is exposed. This fieldmeter measures and records the surface potential on the material as a function of time. The time it takes for the surface voltage to reach $1 / \mathrm{e}(1 / 2.72)$ or $37 \%$ of its maximum value is called the decay time or $\tau$. For materials to possess "good" ESD behavior, they should dissipate the charge faster than one could deposit it in the field. Decay times as short as 0.2 seconds have been quoted for materials to dissipate charge satisfactorily [7], however here we use the criteria that decay times be less than one second.

\section{Spark Incendivity Testing}

If the materials fail any of the above three tests, they will be subjected to a new test based on Spark Incendivity Testing, a new International Standard being created by the International Electrotechnical Commission (IEC) entitled, "Electrostatic Classification of Flexible Intermediate Bulk Containers (FIBC)" [4]. This test method has been successful for evaluating the safety of FIBC which are widely used for the storage, transportation and handling of powdered, flaked or granular material throughout industry. The mishandling, improper grounding, and poor electrostatic dissipation properties of FIBC has led to several hazardous events which have in some cases caused injury and/or death to workers. 
The use of the new IEC test standard should greatly decrease the number of incidents by addressing the ESD hazard properly. Essentially, the test method is to charge a material either by corona charging and/or triboelectric charging and then purposely discharge the surface of the test material (in the form of a spark) using a metal sphere while in the presence of a flammable gas mixture. If the energy of the spark has at least the energy equal to the minimum ignition energy (MIE) of the gas mixture, then the resulting discharge will be capable of igniting the gas mixture. This test method directly measures a material's propensity to deliver sufficient ignitable charge from its surface when a worker and/or object is nearby.

To perform this test, a Spark Incendivity Probe (SIP) is used. A schematic of this probe is shown in Figure 6. Gases with a known MIE enter into a polycarbonate mixing chamber full of glass beads to ensure uniform mixing and to serve as a flame arrestor to prevent back propagation. The gas mixture passes first through fine copper mesh and then into the mixing chamber before passing through a second copper mesh and a perforated metal plate. The gases then surround the electrically grounded brass electrode (with guard) and ignitions occur between the electrode and the charged surface.

The proposed test standard normally calls for gas mixtures of 5.4\% ethylene in air, which has an MIE of $0.14 \mathrm{~mJ}$. This is the MIE of methanol environments that are the worst case for FIBC normally seen in industry. Here however, testing was performed using hydrogen in air at stoichiometric mixtures $\left(30 \% \mathrm{H}_{2}\right.$ and $70 \%$ dry air as before) having a much lower MIE of $0.02 \mathrm{~mJ}$ to represent the worst-case scenario of a charged material in the presence of a hydrogen-enriched atmosphere. Hydrogen and other flammable gases and liquids are commonly used in spaceport operations hence electrostatic charging could pose a problem if incendive discharges exist. Tribocharging occurs in several ways and can be due to charged personnel, charged payloads or equipment, and charged liquids and vapors during typical orbiter processing and operations. 


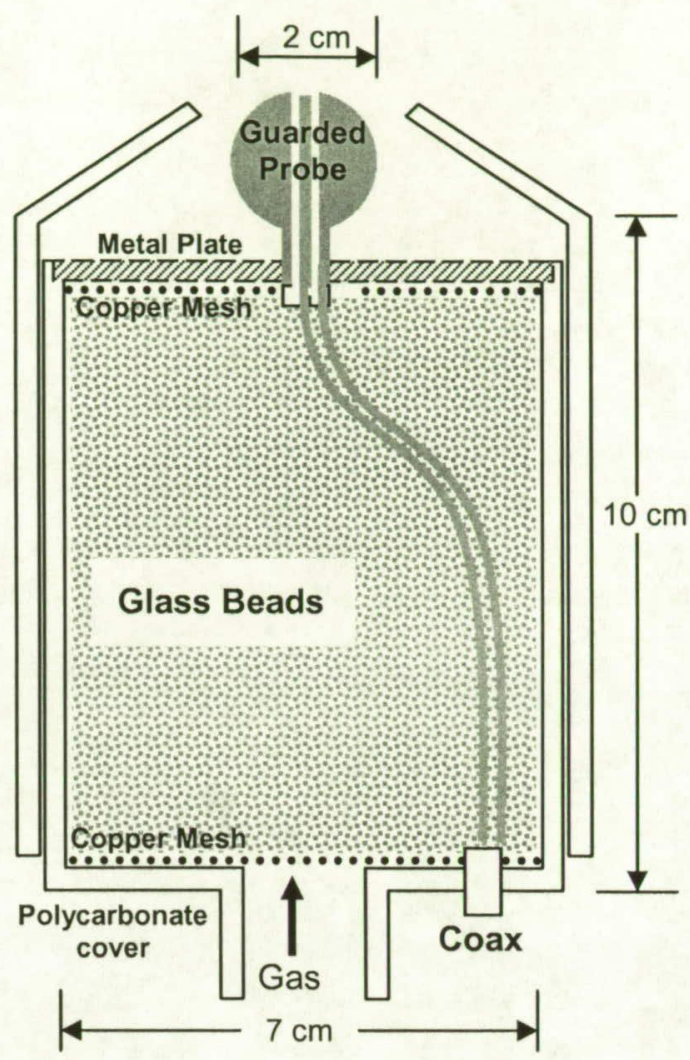

Figure 6: Schematic of the Spark Incendivity Probe [4].

Samples are typically mounted on an insulating frame using clips. Once mounted, the materials were acclimated for up to two days at both $20 \% \pm 5 \%$ relative humidity and $50 \% \pm 5 \%$ relative humidity. Charge was applied to the materials in two ways. The first method was corona charging. A high-voltage power supply was connected to a corona needle plate to creates ions in the air that deposit onto the surface of the test material. The voltage was set to $-30 \mathrm{kV}$ to deposit charge levels similar to what is seen in FIBCs. The second method to apply charge to the materials was by tribocharging with wool. The wool cloth was repeatedly rubbed by hand against the test materials until they were saturated with charge. After the charge deposition onto the surface, the resulting surface potential was measured using a JCI 140 Fieldmeter and recorded. After the test material is fully charged, the gas mixture is allowed to flow through the probe for at least 30 seconds. The probe then approaches the charged material with the speed of approach of about $(0.75 \pm 0.25) \mathrm{m} / \mathrm{s}$. Too slow an approach would cause corona to reduce local charge levels and too fast an approach would cause quenching of the nascent flame kernel. The probe approached the surface in five locations per test and there were at least 25 charging tests giving a total of 
125 approaches per unique environmental condition. Tests were performed at two humidities, using two charging mechanisms and on both sides of the material yielding a total of 1000 tests per sample.

There was simultaneous charge measurements performed during the Spark Incendivity Testing using a guard/electrode geometry. The amount of charge exchanged during electrostatic discharges was measured by connecting the electrode to a large capacitor whose voltage was monitored. In the case that the extracted charge was sufficient to cause an ignition of the hydrogen-air mixture, the ignition event was recorded as a failure.

\section{E. Representative Tests}

Normally, Spark Incendivity is the final step in the selection of a candidate material for use in a possible flammable atmosphere since this method provides sufficient charge that can be extracted from it to create an incendive event. However, if the materials fail this test and no other material is available to replace it, then "representative testing" is required. Unlike Spark Incendivity testing that deposits a significant amount of charge on the test material, representative testing deposits a realistic amount of charge on the materials by simulating its actual usage in the field. If the highest charging mechanism is identified and replicated, then it is possible to experimentally reproduce this scenario whilst immersed in a worst-case flammable atmosphere. If the chargingdischarging of the test materials does not ignite the flammable atmosphere, then and only then can the materials be deemed safe as they are. Otherwise the materials must not be used if a single ignition event occurs. Here each test must be designed and conducted carefully to ensure that the materials are evaluated properly. Tests of this nature require an Incendivity Chamber capable of mounting the test material and reproducing exactly the realistic charging mechanism. The major difficulty with this test method resides in the correct the number of tests to be taken. Too few tests performed results in statistical uncertainties when trying to compute the probability of an incendive event occurrence. Thus it is better to not use the material at all and avoid representative testing if possible. Such discussions are beyond the scope of this paper but these tests have been performed by the ESPL. Results on "Representative Testing" are presented elsewhere [8-10]. 


\section{Results}

Table 1: Test Results of Selected Materials

\begin{tabular}{|c|c|c|c|c|c|c|c|c|c|c|c|c|}
\hline & \multicolumn{2}{|c|}{$\begin{array}{l}\text { Surface Resistivity } \\
\text { (ohms) }\end{array}$} & \multicolumn{4}{|c|}{$\begin{array}{c}\text { Corona Charge Decay Time } \\
(\mathrm{sec})\end{array}$} & \multicolumn{4}{|c|}{$\begin{array}{c}\text { Tribo Charge Decay Time } \\
\text { (sec) }\end{array}$} & \multicolumn{2}{|c|}{ Spark Incendivity } \\
\hline & $50 \% \mathrm{RH}$ & $20 \% \mathrm{RH}$ & $50 \% \mathrm{RH}$ & $P / F$ & $20 \% \mathrm{RH}$ & $\mathrm{P} / \mathrm{F}$ & $45 \% \mathrm{RH}$ & $\mathrm{P} / \mathrm{F}$ & $30 \% \mathrm{RH}$ & $\mathrm{P} / \mathrm{F}$ & $50 \% \mathrm{RH}$ & $20 \% \mathrm{RH}$ \\
\hline Chloroprene & $6.75 E+11$ & $4.19 E+12$ & 0.351 & $P$ & 0.436 & $P$ & 0.454 & $P$ & 1.04 & $\mathbf{P}$ & $\mathrm{P}$ & $\mathbf{P}$ \\
\hline VFH Copolymer & $2.63 E+12$ & $1.34 \mathrm{E}+12$ & 7.96 & $\mathbf{F}$ & 10.2 & $\mathbf{F}$ & 12.9 & $\mathbf{F}$ & 23.1 & $\mathbf{F}$ & $P$ & $\mathbf{P}$ \\
\hline Chlorobutyl-coated Nomex fabric & $3.85 E+12$ & $1.09 E+13$ & $>5 \mathrm{~min}$ & $\mathbf{F}$ & $>5 \mathrm{~min}$ & $\mathbf{F}$ & $>5 \mathrm{~min}$ & $\mathbf{F}$ & $>5 \mathrm{~min}$ & $\mathbf{F}$ & $P$ & $\mathbf{P}$ \\
\hline Bromobutyl & $9.62 \mathrm{E}+12$ & 1.97E+12 & 32.1 & $\mathbf{F}$ & 33.3 & $\mathbf{F}$ & 45.0 & $\mathbf{F}$ & 78.0 & $\mathbf{F}$ & $\mathrm{P}$ & $P$ \\
\hline HDPE fibers & $1.73 E+13$ & $2.40 E+13$ & $>5 \min$ & $\mathbf{F}$ & $>5 \mathrm{~min}$ & $\mathbf{F}$ & $>5 \min$ & $\mathbf{F}$ & $>5 \mathrm{~min}$ & $\mathbf{F}$ & $P$ & $P$ \\
\hline Metallized PTFE 1 & $4.57 E+11$ & $2.80 E+12$ & $>5 \min$ & $\mathbf{F}$ & $>5 \mathrm{~min}$ & $\mathbf{F}$ & $>5 \mathrm{~min}$ & $\mathbf{F}$ & $>5 \mathrm{~min}$ & $\mathbf{F}$ & $\mathbf{F}$ & $P$ \\
\hline Metallized PTFE 2 & $1.52 E+11$ & $5.47 E+11$ & $>5 \min$ & $\mathbf{F}$ & $>5 \mathrm{~min}$ & $\mathbf{F}$ & $>5 \mathrm{~min}$ & $\mathbf{F}$ & $>5 \mathrm{~min}$ & $\mathbf{F}$ & $P$ & $\mathbf{F}$ \\
\hline PTFE-coated fiberglass & $1.20 \mathrm{E}+14$ & $8.70 \mathrm{E}+12$ & $>5 \mathrm{~min}$ & $\mathbf{F}$ & $>5 \mathrm{~min}$ & $\mathbf{F}$ & $>5 \mathrm{~min}$ & $\mathbf{F}$ & $>5 \mathrm{~min}$ & $\mathbf{F}$ & $P$ & $P$ \\
\hline Laminated fabric & $3.20 \mathrm{E}+10$ & $3.28 \mathrm{E}+11$ & 0.27 & $P$ & 0.63 & $P$ & 0.561 & $P$ & 1.0 & $P$ & $P$ & $\mathrm{P}$ \\
\hline polyvinylchloride & $2.40 \mathrm{E}+13$ & $5.04 E+13$ & $>5 \mathrm{~min}$ & $\mathbf{F}$ & $>5 \mathrm{~min}$ & $\mathbf{F}$ & $>5 \mathrm{~min}$ & $\mathbf{F}$ & $>5 \mathrm{~min}$ & $\mathbf{F}$ & $\mathbf{F}$ & $\mathrm{P}$ \\
\hline polycarbonate & $6.32 E+13$ & $1.22 \mathrm{E}+13$ & $>5 \mathrm{~min}$ & $\mathbf{F}$ & $>5 \min$ & $\mathbf{F}$ & $>5 \mathrm{~min}$ & $\mathbf{F}$ & $>5 \mathrm{~min}$ & $\mathbf{F}$ & $\mathbf{F}$ & $P$ \\
\hline nylon & $1.78 E+12$ & $1.06 \mathrm{E}+11$ & 0.593 & $P$ & 1.71 & $\mathbf{F}$ & 1.15 & $\mathbf{F}$ & 2.73 & $\mathbf{F}$ & $P$ & $P$ \\
\hline PTFE-coated fabric & $3.66 \mathrm{E}+11$ & $1.82 E+12$ & 0.167 & $P$ & 1.09 & $\mathbf{F}$ & 0.50 & $\mathbf{F}$ & 2.14 & $\mathbf{F}$ & $P$ & $P$ \\
\hline High density polyethylene & $1.36 \mathrm{E}+13$ & $1.71 \mathrm{E}+13$ & $>5 \mathrm{~min}$ & $\mathbf{F}$ & $>5 \mathrm{~min}$ & $\mathbf{F}$ & $>5 \mathrm{~min}$ & $\mathbf{F}$ & $>5 \mathrm{~min}$ & $\mathbf{F}$ & $\mathrm{P}$ & $P$ \\
\hline Low density polyethylene & $9.15 E+13$ & $1.44 E+13$ & $>5 \mathrm{~min}$ & $\mathbf{F}$ & $>5 \mathrm{~min}$ & $\mathbf{F}$ & $>5 \min$ & $\mathbf{F}$ & $>5 \mathrm{~min}$ & $\mathbf{F}$ & $P$ & $P$ \\
\hline Polyurethane Unmodified & $1.41 E+12$ & - & 0.935 & $P$ & - & - & - & - & - & - & $P$ & $\mathrm{P}$ \\
\hline Polyurethane $3.5 \%$ Ferrocene & $5.40 \mathrm{E}+10$ & - & 0.13 & $\mathrm{P}$ & - & - & - & - & - & - & $P$ & $P$ \\
\hline Polyurethane $10 \%$ Ferrocene & $1.24 E+12$ & - & 0.793 & $\mathbf{P}$ & - & - & - & - & - & - & $P$ & $P$ \\
\hline
\end{tabular}

Table 1 above shows results of the proposed test method for a select number of materials. Only average values are shown in the above data. Standard deviations are not shown. The corona and tribo charge decay values are both listed as the time it takes for the peak voltage to reach $1 / \mathrm{e}$ and not as the time it takes to reach 350 volts, as is done in the pass/fail (P/F) criteria for the triboelectric test. The Spark Incendivity results are listed as combined results of tribocharging and corona charging.

\section{Conclusions}

There are some interesting trends that can be drawn from the data. Only Laminated fabric and polyurethane doped with 3.5\% Ferrocene at high humidity are non-insulators possessing surface resistivities lower than $10^{11} \Omega$. Normally, one expects that insulating properties increases with lower humidity, but there are a few exceptions. Regardless of their insulating characteristics, materials such as the Chloroprene, Laminated fabric 
(at low humidity), nylon and PTFE-coated fabric (at high humidity) show the ability to dissipate applied surface charge. This is further evidence for the occasional lack of correlation between surface resistivity and charge decay. Additionally, charge decay rates for both corona charging and tribocharging are matched well for most materials except for nylon and PTFE-coated fabric in agreement with previous studies [11]. The differences could be accounted for by matching the decay time constants for each test say using $10 \%$ of the initial surface voltage as opposed to an arbitrary $350 \mathrm{~V}$ level.

There were four materials in which ignitions were detected during Spark Incendivity testing. In some of these cases there were multiple ignitions regardless of charging mechanisms. It is interesting to note that a majority of these ignitions occurred during high humidity, which will be discussed further later on. Two materials, the metallized PTFE 1 and the metallized PTFE 2 are metal-backed insulators. They consist of PTFE-coated fiberglass materials backed with a spray deposited aluminum film of different thicknesses. The virgin PTFEcoated material is not susceptible to incendive discharges according to Table 1; however the metal backing provides a termination point for the electric field lines. Now large amounts of charge can be deposited since the field lines are no longer exposed to the air which inherently limits the amount of charge deposited onto an insulating surface due to gas breakdown. The additional charge combined with the charge mobility of the metallic backside creates a dangerous situation susceptible to incendivity discharges. Although the thinness of the insulating material makes propagating brush discharges unlikely to occur, normal brush discharges occur which have sufficient energy to ignite stoichiometric hydrogen-air mixtures. These materials should either not be used or subjected to additional representative testing.

Unfortunately the amount of charge exchanged during a sparking event was not significantly different than that recorded during an incendive event. The reason was determined to be due to the small size of the electrode $(\sim 2$ $\mathrm{mm}$ diameter) used compared to the shield of the probe head. Apparently, each hydrogen ignition created numerous ions that simultaneously shorted the shield to the electrode and thus no appreciable charge was detected. A newer design should be implemented to improve charge measurements. 
The main conclusion from Table 1 is that although materials might posses high surface resistivities and long charge decay times, that does not necessarily indicate that the materials are susceptible to incendive discharges in the presence of hazardous environments. It is perhaps premature to conclude that materials are unsafe electrostatically when measuring only surface resistivity and/or charge decay properties. Equally important is that we have not discovered a case in which materials that pass corona and triboelectric charge decay fail the Spark Incendivity test. Therefore, it is likely that testing surface resistivity and corona and triboelectric charge decay properties alone are more restrictive and are perhaps better served as indicators for more sensitive electrostatic concerns such as those used in microchip and semiconductor manufacturers rather than materials used in flammable atmospheres. However, if materials fail the Spark Incendivity test, they should be tested under representative conditions if the user wishes to continue to use them.

Another important conclusion is that materials may be inherently more dangerous in the presence of higher humidity rather than lower humidity. There have been more ignitions at higher humidity than lower humidity according to the spark incendivity tests. In higher humidities the amount of the charge involved in the discharge is greater due to the increased surface conductivity and larger surface area of charge removed. This is a very important result that seems to contradict the ideology of materials being safe when exposed to higher humidities, environments where charge is able to bleed off more easily. This is the very essence of surface resistivity measurements and charge decay testing. On the contrary, here it is shown that higher humidity may be an unwanted environmental effect for some materials leading to more incendive events even though the surface resistivity and charge decay properties are improved under higher humidity. This is an agreement with Butterworth [12] who discussed that a suitably high resistivity might help avoid incendiary discharges.

The three main conclusions of the proposed test method are that 1) all materials that are slightly conductive should be electrically bonded to ground, 2) materials that are insulating and do not dissipate charge to ground may be intrinsically safe, 3 ) insulating materials may be more susceptible to incendive discharges if exposed to 
high humidities due to increased surface conductivity. In this case Spark Incendivity testing is the only known way to properly evaluate this class of materials since it is able to differentiate between intrinsically safe insulators and those inherently susceptible to incendive electrostatic discharges.

\section{Acknowledgements}

We would like to acknowledge the Joint ESD Working Group led by Kreg Rice of Boeing at the NASA Johnson Space Center and Chilworth Technologies for providing essential guidance in the design of the overall test methodology and providing representative thermal blankets. We thank John Chubb of John Chubb Instruments for useful discussions and Trent Smith for providing samples.

\section{References}

[1] Finchum, A., "Standard Test Method for Evaluating Triboelectric Charge Generation and Decay", KSC Test Standard MMA-1985-98, Revision 3 (1998).

[2] Taylor, D.M. and Elias, J., "A versatile charge decay meter for assessing antistatic materials", Institute of Phys. Conf. Ser. No. 85 Section 2, Electrostatics '87, Oxford (1987).

[3] Fowler, S., "Surface Resistivity and Static Decay Do Not Correlate", Proc. EOS/ESD Symp., pp. 7, (1989).

[4] International Electrotechnical Commission, "Part 4-4: Standard test methods for specific applications Electrostatic classification of flexible intermediate bulk containers (FIBC)", IEC 61340-4-4:2005, (2005).

[5] ESD Association Standard Test Method ESD STM11.11, "Surface Resistance Measurements of Static Dissipative Planar Materials", (2001).

[6] British Standard BS 7506, Methods for Measurments in Electrostatics, Part 2 (1995).

[7] Chubb, J., "Measurement of Tribo and Corona Charging Features of Materials for Assessment of Risks from Static Electricity", IEEE Transactions on Industrial Applications, vol. 36, no. 6, (2000).

[8] Buhler, C.R. and Calle, C.I., "Electrostatic Evaluation of the STS Thermal Control System Multilayer Insulation Blankets", NASA/TM-2004-211521 (2004). 
: [9] Buhler, C.R. and Calle, C.I., "Final Results of the Electrostatic Evaluation of the STS Thermal Control System Multi-Layer Insulation Blankets", submitted as a NASA/TM 2005-211542 (2005).

[10] Buhler, C.R. and Calle, C.I., "Results of the Electrostatic Evaluation of the ISS Thermal Shrouds and the ISS Multi-Layer Insulation Blankets", submitted as a NASA/TM 2005-211541 (2005).

[11] Gomph, R., Holdstock, P., and Chubb, J.N., "Electrostatic Test Methods Compared", EOS/ESD Symposium, September 26-30, 1999.

[12] Butterworth, G.J., Paul, E.S., and Chubb, J.N., "A study of the incendivity of electrical discharges between planar resistive electrodes", 'Electrostatics 1983', IoP Confr Series, 66 p185 (1983). 